\title{
Averaging hydraulic head, pressure head, and gravitational head in subsurface hydrology, and implications for averaged fluxes, and hydraulic conductivity
}

\author{
G. H. de Rooij \\ Wageningen University, Centre for Water and Climate, Soil Physics, Ecohydrology and Groundwater Management, \\ Wageningen, The Netherlands
}

Received: 9 April 2008 - Published in Hydrol. Earth Syst. Sci. Discuss.: 9 May 2008

Revised: 28 May 2009 - Accepted: 2 July 2009 - Published: 13 July 2009

\begin{abstract}
Current theories for water flow in porous media are valid for scales much smaller than those at which problem of public interest manifest themselves. This provides a drive for upscaled flow equations with their associated upscaled parameters. Upscaling is often achieved through volume averaging, but the solution to the resulting closure problem imposes severe restrictions to the flow conditions that limit the practical applicability. Here, the derivation of a closed expression of the effective hydraulic conductivity is forfeited to circumvent the closure problem. Thus, more limited but practical results can be derived. At the Representative Elementary Volume scale and larger scales, the gravitational potential and fluid pressure are treated as additive potentials. The necessary requirement that the superposition be maintained across scales is combined with conservation of energy during volume integration to establish consistent upscaling equations for the various heads. The power of these upscaling equations is demonstrated by the derivation of upscaled water content-matric head relationships and the resolution of an apparent paradox reported in the literature that is shown to have arisen from a violation of the superposition principle. Applying the upscaling procedure to Darcy's Law leads to the general definition of an upscaled hydraulic conductivity. By examining this definition in detail for porous media with different degrees of heterogeneity, a series of criteria is derived that must be satisfied for Darcy's Law to remain valid at a larger scale.
\end{abstract}

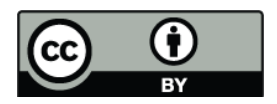

Correspondence to: G. H. de Rooij (ger.derooij@wur.nl)

\section{Introduction}

In saturated and unsaturated water flow in porous media, the continuum approach is generally invoked (e.g., Bear and Bachmat, 1991), and the resulting laws of mass conservation and movement, as well as the variables appearing in there, are defined for a mathematical point centered within a Representative Elementary Volume (REV). The resulting equation for water movement is Darcy's Law. Its general acceptance is illustrated by the fact that the REV-scale has frequently been referred to as the Darcy scale (e.g., Bhattacharya and Gupta, 1983; Jacquin and Adler, 1987; Kavvas, 2001, among many others).

In order to develop theories (and their associated measurable variables) at scales that better correspond to the scale at which typical real-world problems manifest themselves, there is a drive to upscale these REV-scale relationships and variables to larger spatial scales. In doing so, pertinent questions arise as to the physical soundness of upscaling variables such as fluid pressure, matric and hydraulic potential, and water flux. It must be stressed that this problem differs from upscaling operation that gives rise to the REV-scale description of flow in porous media from pore scale phenomena. At the pore scale, phases are discretely distributed, phase properties are only defined in locations where the phase is present, and the interaction between phases is governed by phase interfaces and the associated surface tensions (see Gray and Miller, 2007, for an overview), and exchange of mass and momentum across the interfaces (Raats and Klute, 1968a, b). At the REV-scale, phase distributions are represented by volume fractions, interfacial effects are described in terms of pressure differences between phases, and displacement of phases is described by changes of the volume fractions the

Published by Copernicus Publications on behalf of the European Geosciences Union. 
phases occupy and by flux densities over cross-sectional areas spanning many pores. These properties are obtained by volume-averaging the pore-scale properties. The averaging theorem, (e.g., Whitaker, 1967; Gray, 1975; Whitaker, 1986) attributed to various authors (Howes and Whitaker, 1985), specifically deals with the values at interfaces of given quantities to aid in determining the volume average of the gradient of such quantities. The theorem was questioned by Veverka (1981) and refined by Howes and Whitaker (1985), but both agree that for conditions normally found in flow in porous media, the theorem holds.

In the next-larger upscaling operation, from the REV-scale to more practically relevant scales, the continuum approach is preserved (thereby eliminating the problems associated with phase interfaces), and the key point is the incorporation of spatial variations in volume fractions, flux densities, and pressures in the larger-scale formulation. The need to describe flows in porous media at scales beyond the REVscale has been well recognized and has prompted various lines of attack (see Renard and de Marsily (1997) and Kitanidis (1997) for an overview of methods to upscale saturated hydraulic conductivities, Harter and Hopmans (2004) and Vereecken et al. (2007) for upscaling flows and flow parameters in unsaturated soils). To review these in detail would be beyond the scope of this paper. A common feature is that tractable solutions of the upscaling problem require that limitations be imposed to the allowable degree of heterogeneity, the nature of the random field representing the spatial variation of the porous medium, or both. In addition, only specific megascopic (i.e. larger than the REV-scale) flow patterns may be permitted. Limitations regarding the degree of variation are severe given the extreme variability of porous medium properties (Jury et al., 1991; p. 269), in particular the hydraulic conductivity at saturation. In the unsaturated zone, the hydraulic conductivity can vary over many orders of magnitude. The assumption of (log-)Gaussianity of soil hydraulic properties is frequently invoked, but hardly ever tested. De Rooij et al. (2004) concluded that higher-order moments should not be neglected.

Often, the dimensions of the megascopic flow field of interest are defined by aquifer bounds, horizontal dimensions of agricultural fields or natural areas, and the vertical extent of the soil. These dimensions often are too small to validate the assumption of ergodicity, particularly in soils where the spatial extent in the megascopic (vertical) flow direction is generally limited (e.g., Yeh, 1998). In addition, subsurface hydraulic properties vary over many scales. Few data sets are available that allow an investigation of the spatial nature of such variations, but the assumption that distinct hierarchic scales exist at which heterogeneities present themselves is not axiomatic. The only scale at which this scale hierarchy has been deployed with some success is between the pore scale and the REV-scale. Still, even here it is likely that the REV-size depends on the parameter of interest (Bear and Bachmat, 1991). At larger scales, sedimentary and pedogenetic processes make it more likely that, rather than a clear scale hierarchy, there exists a scale continuum at which spatial variation manifests itself. Again, relatively small volumes of interest (agricultural fields, units on a soil map, aquifers consisting of buried beds of paleorivers) aggravate the effect on the acceptability of the various assumptions needed.

The focus of this paper is primarily on upscaling from the REV-scale through averaging over volumes and crosssections beyond the scale of the REV. The technique of volume averaging beyond the REV-scale was developed elaborately by Quintard and Whitaker (1988) (from here on denoted as QW) and more schematically by Gray and Miller (1984). Since the interest is predominantly in the upscaling of Darcy's Law we do not delve into the thermodynamic constraints that are being developed in an on-going series of papers (e.g., Jackson et al., 2009, and references therein). The analysis of QW is sophisticated but requires numerous simplifying assumptions. The value of QW's work is enhanced by their careful identification of these assumptions and simplifications that are required to obtain the desired volume averages at the large scale (Sect. 2 in QW) and solve the closure problems that arise from the need to express the variations within the averaging volume in terms of the averaged values. The most pertinent of those assumptions and simplifications are discussed in the following, not to criticize the work of QW but to assess for which types of problems alternative approaches may need to be developed. In following sections, some of the assumptions are relaxed to find results that are valid for a wider range of cases. This is done at the cost of mathematical finesse: for instance, local variations can no longer be expressed in terms of the averages. As a consequence, closure will no longer be possible. Because the paper is of considerable length, equations and pages in QW are referenced where needed.

\subsection{Assumptions and simplifications in volume averaging}

\subsubsection{The necessity of a scale hierarchy}

In QW Eq. (2.28) and the text below it, the pore scale (microscopic) is identified as the smallest and the reservoir scale (gigascopic scale) as the largest of four length scales. The second scale is the REV-scale (macroscopic scale), and the third is the scale (megascopic scale) addressed by large-scale averaging; these are the scales of interest here. Every scale must be much larger than the previous, but how much larger is not specified. However, the measures of scale figure regularly in order-of- magnitude terms that often need to be negligible in the subsequent derivations. Therefore, a minimum of two orders of magnitude difference between subsequent scales seems reasonable. With the REV-scale in the order of $0.1 \mathrm{~m}$, that makes the megascopic scale in the order of $10 \mathrm{~m}$, which is acceptable in the horizontal direction 
for many aquifers but already too large for the vertical direction in aquifers with sedimentary layers and many unsaturated zones.

\subsubsection{The requirement of spatially periodic boundary conditions}

The spatially periodic boundary conditions (forced upon QW to keep the problem mathematically tractable) limit the application of the approach to small systems, although Crapiste et al. (1986) argue that periodic boundary conditions are not necessary for all problems. The limitations of the use of periodic boundary conditions imposed on a repetitive unit cell that represents the porous medium were put in perspective by Renard and de Marsily (1997) by noting that large-scale parameters should not depend on the boundary conditions, and that the unit cell should be small compared to the flow domain of interest. But if the unit cell at the megascopic scale must be in the order of $10 \mathrm{~m}$ (see above), the latter condition cannot always be met. Furthermore, in soils, the root zone is of particular interest, and mass exchange between the soil and the atmosphere across the soil surface is key; such fluxes will never be periodic with increasing depth. The periodic boundary conditions also seem to implicitly limit the application to megascopically unidirectional flow. Flows toward wells in aquifers are clearly incompatible with that requirement. Finally, periodic boundary conditions are difficult to reconcile with transient flows.

\subsubsection{Limitation to quasi-steady flow}

Another factor affecting the applicability to non-steady conditions is the fact that the closure problem does not include the effect of moving contact lines (where phase interfaces meet), as indicated below Eq. (1.16) in QW. For unsaturated flows, this means that only quasi-steady flows can be considered, leaving problems involving infiltration beyond reach of the analysis (QW, p. 392). But even for saturated flows, QW consider the dynamic problem insolvable at this time because expressions for the dynamic megascopic-scale momentum equations cannot be found (QW, p. 393). Note that the upscaling operation itself necessarily increases the characteristic time scale of the upscaled equations, and that highly dynamic situations lend themselves poorly to upscaling (Roth, 2008).

\subsubsection{Decomposition of spatial variation}

It is assumed throughout in QW, but also in other papers (e.g., Gray, 1975; Crapiste et al., 1986; Whitaker, 1986) that local values (within the averaging volume) of a quantity can be decomposed into its intrinsic phase average (a volume average over the volume occupied by the phase of interest) and a zero-averaged deviations. This decomposition relies on the disjoint hierarchical sequence of scales, even though there is no formal requirement for the spatial structure of the deviations. But in a porous medium with a continuum of spatial scales for heterogeneities, it is very difficult to arrive at an unambiguous value of the intrinsic phase average since it will depend on the size of the averaging volume on every length scale. This in turn leaves the deviations poorly definable. In particular, this can be problematic at often blurred boundaries between sedimentary layers or soil horizons.

There are more subtle limitations and assumptions at play, but these do not seem to dramatically alter the conclusion that can be drawn from considering the assumptions above: for flow problems that are decidedly transient, and/or occur in media without clear scale hierarchy of its spatial variation in at least one direction, and/or involve a system boundary, upscaling by volume averaging and solving the closure problem at this time is unlikely to generate meaningful results. This is corroborated by attempts by Quintard and Whitaker (1990a, b) and Bertin and Quintard (1990) to tackle theoretically, numerically, and experimentally problems that try to relax some of the limitations imposed by QW. The resulting equations were vastly more complex and still were unable to handle situations that involved ordinary imbibition and drainage processes and included gravity (Quintard and Whitaker, 1990a). Roth (2008) pointed out that averaging flow over larger areas and volumes increases the characteristic time scale of the equation describing the large-scale flow. This creates a fundamental obstacle for volume-averaging highly transient processes (most notably infiltration with a sharp wetting front).

\subsection{Gravity}

At the Darcy- (macroscopic) scale and the megascopic scale, the various forces and pressures acting in the pores are efficiently unified in the core-scale matric and gravitational potential while the kinetic energy can generally be ignored. Whatever the method of upscaling may be, gravity gains importance as the vertical dimension of the system under study expands (QW (p. 403) indicated that gravitational effects had to be negligible for the closure calculations to be tractable in their test problems of vertically stratified media and highly schematized two-dimensional spatially periodic media). In natural systems, gravity manifests itself in such phenomena as the finite height of capillary rise in the unsaturated zone, and in fluid pressure in the saturated zone.

The role of gravity and pressure is apparent in the work of Gray and Miller (2004), who studied the upscaling of Darcy's Law in an idealized system involving a configuration with horizontal flow in a volume containing two porous media with different porosities. Gray and Miller (2004) averaged over a cross-section perpendicular to the main direction of flow, thereby bypassing the difficulties associated with averaging fluxes over volumes (Nordbotten et al., 2007). They reported inconsistencies in Darcy's Law if the layering of the media was tilted. Their analysis also discussed the averaging of fluid pressure. In the unsaturated zone, the matric 
potential is the equivalent to fluid pressure in the saturated zone. Therefore, the work of Gray and Miller (2004) may also have ramifications for averaging the matric potential of a soil volume, and thus for the determination of soil water retention curves at various scales. The effect of gravity on the nature of the soil water retention curve was experimentally addressed in another context by Liu and Dane (1995), and their findings will help clarify the issues raised by Gray and Miller (2004).

\subsection{Alternative approach}

In view of the limitations identified above associated with upscaling by volume averaging, the problem is attacked in a way that invokes as few assumptions as possible. Clearly, the worthy pursuit of a megascopic flow equation with megascopic parameters then becomes untenable. On the other hand, more can be learned from the way in which spatial variation affects the range of validity of Darcy's Law by developing expressions that allow any type of variation to be traced and its effect quantified. From generally accepted physical realities, a volume averaging formalism is derived that is proved to resolve the inconsistencies reported by Gray and Miller (2004) between formulations of Darcy's Law and its upscaled equivalent, which they illustrated for a special case. The formalism will be used to develop an area-averaged formulation of Darcy's Law, which is used to examine the conditions for which the REV-scale version remains valid under the averaging operation.

\section{Theory}

\subsection{The potential energy of subsurface water}

A solid foundation of the potential of subsurface water is conducive to a proper treatment of the various potentials during upscaling operations. Groenevelt and Bolt (1969) were among the first to analyze flow through porous media within the framework of non-equilibrium thermodynamics. They declared the pressure a potential that was additive to the gravitational potential, which can be formalized as

$\psi=p+\rho g\left(x_{3}-x_{3, \text { ref }}\right)$

where $\psi$ is the total potential per volume $\left[\mathrm{ML}^{-1} \mathrm{~T}^{-2}\right], p$ is the fluid pressure $\left[\mathrm{ML}^{-1} \mathrm{~T}^{-2}\right], \rho$ is the fluid density $\left[\mathrm{ML}^{-3}\right]$, $g$ is the gravitational acceleration $\left[\mathrm{LT}^{-2}\right], x_{3}$ is the vertical coordinate [L] (positive upward), and $x_{3, \text { ref }}[\mathrm{L}]$ is an arbitrary reference height. Note that the inclusion of $x_{3 \text {,ref }}$ makes the potential relative. Since the gradient of $\psi$ is all that is needed, this is inconsequential.

The superposition of the potentials created by the pressure field and by the gravitational field suggests that methods developed to average one potential should be applicable to the other as well. Furthermore, the superposition principle implies that, in any upscaling operation through systematic averaging, the averaging manipulations must be identical for both potentials to maintain the superposition property across

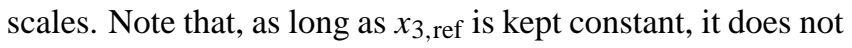
impair the superposition property.

In subsurface hydrology, the hydraulic and matric head are related as (e.g., Brutsaert, 2005; Jury et al., 1991):

$H=h+\left(x_{3}-x_{3, \text { ref }}\right)$

It is immediately clear that this textbook equation can be obtained by dividing the thermodynamically established Eq. (1) by $\rho g$ and replacing $p$ by $p$ minus the atmospheric pressure to allow $h$ to be zero at the phreatic level. In this way the pressure is made relative in the same fashion as the elevation in Eq. (1). Without loss of generality we can set the arbitrary

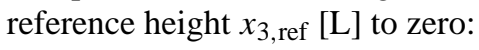

$H=h+x_{3}$

In a multiphase system (solid-fluid-gas) with the gas phase at instantaneous equilibrium with the atmosphere, the pressure in the fluid exceeds the atmospheric pressure when $h \geq 0$, and $h$ is routinely termed the pressure head. For wetting fluids this implies that the medium is saturated with this fluid. For matric potentials $<0$, the fluid pressure is smaller than atmospheric pressure, and $h$ represents the matric and interfacial forces that retain the fluid in the pores. It is therefore termed matric head. Note that the magnitude of the matric forces can cause values of $h$ that are so low that the pressure equivalent would be negative. Since negative pressures are physically unacceptable, Hassanizadeh and Gray (1990) proposed a wettability potential in addition to the fluid pressure to represent the effect of matric and interfacial forces on a phase. This wettability potential quantifies the energy change of the phase associated with a change in saturation. The sum of the fluid pressure potential (relative to the atmospheric pressure potential) and the wettability potential gives $h$. The wettability potential satisfies the superposition property of the pressure and the gravitational potential. Therefore, the remainder of the text simply uses $h$. The decomposition into the contributing potentials is straightforward.

The excessive pressure of the air for $h<0$ allows it to invade the pores. The extent to which it will do so is described by the fluid retention curve, which indicates how much fluid resides in the pore space at a given matric head. Thus, this generally hysteretic curve, which is specific for a combination of medium and fluid, facilitates a consistent characterisation of the system in terms of fluid content and energy status of the fluid (Jury et al., 1991; Hillel, 1998).

\subsection{Upscaling by spatial averaging}

The discussion here pertains to upscaling within a soil volume that is larger than the REV. In the analysis, we only require that the REV exists, that the flow is laminar everywhere (thus ensuring the validity of Darcy's Law at this 
scale), that the gas phase pressure is always at equilibrium with the atmosphere, and that the fluid is incompressible and has a constant density. As discussed above, the existence of the REV implies that the volume-averages of the geometrical characteristics of the microscopic structure of the pore space and the phase distribution within it do not depend on the size of the REV, and therefore the characteristic length scale of these variations must be much smaller than that of the REV (see Bear and Bachmat, 1991). But similar constraints on the separation between the REV-scale and the next-higher averaging scale are not invoked: the averaging volume may be large enough to be heterogeneous in a nonstationary sense (Cressie, 1993), i.e., trends in soil hydraulic properties may be present. There may even exist a continuous range of scales at which variations in hydraulic properties of the porous medium manifest themselves.

The following focuses on upscaling Darcy's Law and examining the conditions for which it retains its validity beyond the REV-scale. Therefore, the closure problem will not be addressed, which considerably simplifies the mathematical intricacies of the analysis. This is why the imposed constraints can be much more flexible than those typically deployed in the volume averaging literature (see the Introduction). There, the desire to solve the closure problem necessitated a more stringent set of limitations to keep the problem tractable. As a consequence, the analysis can address the continuum of scales beyond the REV-scale, instead of the discrete scale jumps that were explored by Gray (1975), Crapiste et al. (1986), and QW, among others.

As indicated above, the validity of Darcy's Law at the REV-scale is not disputed:

$\boldsymbol{q}=-\mathbf{K} \nabla H$

where $\boldsymbol{q}\left[\mathrm{LT}^{-1}\right]$ is the volumetric flux density vector and $\mathbf{K}$ $\left[\mathrm{LT}^{-1}\right]$ is the hydraulic conductivity tensor.

The focus is on water flow in porous media; below, $\boldsymbol{x}$ denotes the location vector, with $x_{1}$ and $x_{2}$ the horizontal coordinates [L], $x_{3}$ the vertical coordinate as before [L], and $t$ time [T]. The variables of interest are the porosity $n(x)$, the volumetric water content $\theta(\boldsymbol{x}, t), h, H, \boldsymbol{q}$ (all functions of $\boldsymbol{x}$ and $t$ ), and $\mathbf{K}(\boldsymbol{x}, \theta)$. For a saturated porous medium, $\mathbf{K}(\boldsymbol{x}, \theta)$ simplifies to $\mathbf{K}(\boldsymbol{x})$. By letting $n$ vary in space but not in time we exclude from the analysis porous media with varying pore space (i.e., swelling and shrinking soils), and implicitly limit the discussion to time scales much smaller than those of interest for most geological and soil morphological processes. See Raats and Klute (1968a, b) for the treatment of soils with a non-rigid matrix.

Large-scale averages of porosity and volumetric water content are easily found and their physical meaning is immediately clear:

$n_{V}=\frac{1}{V} \int_{V} n \mathrm{~d} V$
$\theta_{V}=\frac{1}{V} \int_{V} \theta \mathrm{d} V$

where $V\left[\mathrm{~L}^{3}\right]$ denotes an arbitrary volume occupied by a porous medium, and the subscript $V$ denotes the volumeaveraged value of the subscripted variable. Note that Eqs. (5) and (6) are themselves upscaled values of volume averages of indicator functions that take on the value of 1 whenever they are located in a pore or in the water phase, respectively (see also Nordbotten et al., 2007). The main difference is observational: measuring $n$ and $\theta$ is feasible in realistic porous media, while the indicator functions can only be determined in small, simplified systems. Averages of $n$ and $\theta$ over an arbitrary area or line along the principal directions within the porous medium can be found by a corresponding reduction of the dimensions over which the integrals in Eqs. (5) and (6) are performed.

As shown above, the hydraulic head represents the total energy of the water at a given location. Its spatial average should therefore reflect the total energy of the water present in the porous medium volume for which the average is determined. Therefore, the local values should be weighted by the local water amount. With $H$ being the energy per unit weight (Hillel, 1998), weighting by the local weight $(\theta \rho g)$ would be consistent. But since $\rho g$ is assumed constant, this simplifies to weighting by $\theta$ :

$H_{V}=\frac{\int_{V} \theta H \mathrm{~d} V}{\int_{V} \theta \mathrm{d} V}$

with the subscript $V$ denoting a volume average as above. The weighting by $\theta$ ensures that Eq. (7) satisfies the additivity condition advocated by Gray (2002) in that it conserves the total energy irrespective of the size of $V$.

Because of the identical averaging manipulations required by the superposition property of the components $h$ and $x_{3}$ of $H$ and, we also have:

$h_{V}=\frac{\int_{V} \theta h \mathrm{~d} V}{\int_{V} \theta \mathrm{d} V}$

and

$x_{3, V}=\frac{\int_{V} \theta x_{3} \mathrm{~d} V}{\int_{V} \theta \mathrm{d} V}$

Equation (9) identifies the horizontal plane around the center of gravity of the water in $V$. Note that the numerators of Eqs. (7-9) have dimensions $\mathrm{L}^{4}$ and are measures of the total, matric, and gravitational energy, respectively, stored in a body of subsurface water at a given time. If desired, the relative nature of $H, h$, and $x_{3}$ can be removed, and the resulting absolute values can be multiplied by $\rho g$ to obtain the respective energies in Joules. 
Note that Eq. (8) differs from earlier averaging procedures by Lee et al. (2007) and Zehe et al. (2006), who took the volume-average of the matric potential over the entire averaging volume, i.e., the solid phase, the gas phase, and the liquid phase within $V$. In view of the above, this leads to a systematic underestimation of $h_{V}$, because under local equilibrium, the lower (more negative) values of $h$ will be associated with lower water contents and therefore should be assigned smaller weighting factors to satisfy the requirement that energy is conserved in the volume-averaging operation.

As with $n$ and $\theta$, averages of the various potentials over an arbitrary area or line along the principal directions within the porous medium can be found by modifying the dimensions over which the integrals in Eqs. (7-9) are performed. In many cases, a flow has a well-defined megascopic direction, for instance because the flow domain is enclosed by impermeable barriers on all but two opposite sides. Integrations over cross-sections perpendicular to the megascopic flow direction can then provide average potentials that are local with respect to the coordinate in the flow direction. This is particularly useful for calculating upscaled head gradients.

Any of the tensorial components of $\mathbf{K}$ can be volumeaveraged, but the resulting average does not bear a relationship to any flux at the scale of $V$. For $\mathbf{K}$ this direct approach seems to be of limited value. Averaging the components of $\boldsymbol{q}$ is more fruitful. Here, volume averages are less useful (as Nordbotten et al., 2007 also noted) than averages over some area (e.g., the boundary of $V$, or a cross-section through $V$ ). This is a direct consequence of the applicability of Green's divergence theorem to relate the mass change in a volume to the mass flux across its boundary (see Raats and Klute, 1968a, b, among others). For simplicity, we limit the discussion to averages over areas in two principal directions. In that case, the flux component $q_{j}\left[\mathrm{LT}^{-1}\right](j \in\{1,2,3\})$ in the remaining principal direction is of interest, and its average $q_{j, A}\left[\mathrm{LT}^{-1}\right]$ over area $A\left[\mathrm{~L}^{2}\right]$ in the directions of $x_{i}$ and $x_{k}$ $(i \in\{1,2,3\}, k \in\{1,2,3\}, i \neq j \neq k)$ is:

$q_{j, A}=\frac{1}{A} \int_{A} q_{j} \mathrm{~d} A=-\frac{1}{A} \int_{A} K_{j} \frac{\partial H}{\partial x_{j}} \mathrm{~d} A$

where the off-diagonal elements of $\mathbf{K}$ were assumed to be equal to zero. The notation $K_{j j}$ was therefore simplified to $K_{j}$. Note that the averaged flux density involves areal averages instead of volume averages. By calculating an areal average hydraulic head at two planes of area $A$ and located at $x_{j}-\Delta x_{j} / 2$ and $x_{j}+\Delta x_{j} / 2$, and taking the limit as $\Delta x_{j} \downarrow 0$, the areally averaged hydraulic gradient is obtained:

$$
\frac{\partial H_{A}}{\partial x_{j}}=\frac{\partial}{\partial x_{j}} \frac{\int_{A} \theta H \mathrm{~d} A}{\int_{A} \theta \mathrm{d} A}
$$

The averaged flux density and hydraulic gradient can be combined in a form that is analogous to Darcy's Law:

$q_{j, A}=-K_{j, A} \frac{\partial H_{A}}{\partial x_{j}}$

The definition of the upscaled hydraulic conductivity $K_{j, A}$ $\left[\mathrm{LT}^{-1}\right]$ follows immediately:

$K_{j, A}=\frac{1}{A} \int_{A} K_{j} \frac{\partial H}{\partial x_{j}} \mathrm{~d} A\left[\frac{\partial}{\partial x_{j}}\left(\frac{\int_{A} \theta H \mathrm{~d} A}{\int_{A} \theta \mathrm{d} A}\right)\right]^{-1}$

Time does not appear in this equation because the assumption of immediate equilibrium between $h$ and $\theta$ that underlies Richards' equation is adopted. Still, this is a less severe restriction than the requirement of non-moving contact lines discussed in Sect. 1.1.3. As is to be expected, the upscaled version of the hydraulic conductivity in Eq. (13) generally behaves in a non-Darcian way. Since the numerator and the denominator are not proportional, the upscaled hydraulic conductivity will depend on the magnitude of the upscaled hydraulic gradient. Furthermore, an infinite number of configurations of local values of $\theta$ and $H$ exist that will yield the same integrals in the denominator of Eq. (13) but, through the different local values of $K_{j}$, different values of the upscaled hydraulic conductivity. Thus, $K_{j, A}$ is not unique for a given value of $\partial H_{A} / \partial x_{j}$.

\subsection{Criteria for determining the validity of Darcy's Law at a given scale}

It should be noted that Eqs. (10), (11), and (13) preserve the local values of $\theta, H$, and $K_{j}$. Thus, they allow one to insert any spatial distribution of these values (obtained by measurement, simulation, or any other suitable means) and investigate, for various values of $A$, the behavior of the upscaled flux density, hydraulic gradient, and hydraulic conductivity. To do so here is outside the scope of the paper, but the form of the equations permits the inference of favorable conditions for the use of Darcy's Law at larger scales.

Specifically, two requirements need to be met to make Darcy's Law valid for an area $A: K_{j, A}$ should not vary with the flux density $q_{j, A}$ across $A$, and $K_{j, A}$ should be unique for any gradient $\partial H_{A} / \partial x_{j}$. The validity criterion following from the first requirement is:

$$
\frac{\int_{A} K_{j} \frac{\partial H}{\partial x_{j}} \mathrm{~d} A}{\frac{\partial}{\partial x_{j}}\left(\frac{\int_{A} \theta H \mathrm{~d} A}{\int_{A} \theta \mathrm{d} A}\right)} \approx C
$$

with $C\left[\mathrm{LT}^{-1}\right]$ a constant. For saturated porous media, $C$ is a true constant that should not vary in time. For unsaturated porous media, the value of $C$ depends strongly on the degree of saturation. Note that the criterion is relatively flexible in that it only involves integrations of various properties. 
But the uniqueness requirement, which is less easily captured in a similar form, imposes limits on the permitted degree of variation within $A$ of the various integrands.

Equation (14) is a very general criterion. For various restrictions on the degree of variation of some of the characteristics of the flow, it can be simplified. For instance, if $\theta$ varies little over $A$, Eq. (13) simplifies to (after invoking Leibniz' rule):

$K_{j, A}=\frac{\int_{A} K_{j} \frac{\partial H}{\partial x_{j}} \mathrm{~d} A}{\int_{A} \frac{\partial H}{\partial x_{j}} \mathrm{~d} A}$

and the corresponding validity criteria (for $K_{j, A}$ being independent of the gradient) become:

$$
\frac{\int_{A} K_{j} \frac{\partial H}{\partial x_{j}} \mathrm{~d} A}{\int_{A} \frac{\partial H}{\partial x_{j}} \mathrm{~d} A} \approx C, \quad \frac{\theta_{\max }-\theta_{\min }}{\theta_{A}} \ll 1
$$

where $\theta_{A}$ is the areal average of $\theta$ within $A$, and $\theta_{\min }$ and $\theta_{\max }$ are its minimum and maximum value, respectively. Here too, $C$ is a true constant when the porous medium is saturated and will depend on the degree of saturation if the medium is not. The requirement of limited variations in $\theta$ can be satisfied for relatively large $A$ in an aquifer (segment) of relatively uniform composition, and in dry soils, where the local $\theta$ hardly depends on $h$ (steep end of the soil water characteristic), and $\theta$ at a given $h$ is essentially determined by soil texture (e.g., Hillel, 1998). Equation (15) indicates that the upscaled hydraulic conductivity is the average of the local conductivities weighted by the local hydraulic gradients. Since the local hydraulic gradients affect the field of local $H$-values, they indirectly affect the distribution of local $\theta$-values through the soil water characteristic. Consequently, $K_{j, A}$ is still dependent on $\partial H_{A} / \partial x_{j}$. For saturated flow this limitation does not apply, and furthermore, $K_{j, A}$ is not affected by a scalar multiplication of the field of $\nabla H$. Although different distributions of $\partial H / \partial x_{j}$ can still produce the same $\partial H_{A} / \partial x_{j}$ but different $q_{j, A}$, this non-uniqueness risk is probably limited for problems in which the megascopic flow direction does not change too much.

For dissipative $H$ (little variation across $A$ in $H$ and $\left.\partial H / \partial x_{j}\right)$, Eq. (13) reduces to:

$K_{j, A}=\frac{1}{A} \int_{A} K_{j} \mathrm{~d} A$

with the associated validity criteria:

$$
\frac{H_{\max }-H_{\min }}{H_{A}} \ll 1, \frac{\frac{\partial H}{\partial x_{j}} \max -\frac{\partial H}{\partial x_{j}} \min }{\frac{\partial H_{A}}{\partial x_{j}}} \ll 1
$$

With the weighting by local gradients removed, $K_{j, A}$ for saturated flow becomes both unique and independent of the flux density, and the upscaled Darcy's Law can be applied as long as the above conditions are met, which is the case if $\partial H / \partial x_{j}$ remains below a critical level everywhere. This condition can be met if the flux density integrated over the aquifer thickness is small compared to the aquifer's transmittivity. For the unsaturated zone, local conductivity values can vary strongly even if the matric potential varies relatively little. Hence, even though $K_{j}$-values are not weighted, the possibility of non-uniqueness remains.

It is important to note that in all criteria the hydraulic conductivity (the most variable parameter of the subsurface) remains variable. Furthermore, none of the criteria imposes implicit bounds on the nature or the degree of variability of $K_{j}$.

If $A$ is oriented horizontally during unit gradient conditions in the unsaturated zone, the flow is perfectly vertical everywhere, with $H$ constant across $A$, and $\partial H / \partial x_{3}=1$ everywhere. Under these conditions, the requirements of Eq. (18) are satisfied, Eq. (17) is valid and the upscaled Darcy's Law (Eq. 12) can be used with $\partial H_{A} / \partial x_{3}=1$. When the groundwater level is deep (at least several meters) and a thick nonlayered soil horizon exists, unit gradient conditions can prevail over long time periods in this horizon, and the $K_{j, A}$ then is equal to the long term net infiltration (see Wagenet, 1986).

Highly transient conditions in unsaturated zone lead to strongly varying values of $K_{j}$ and the two spatial gradients in Eq. (13). As a consequence, the dependence of $K_{j, A}$ on $\partial H_{A} / \partial x_{j}$ and the non-uniqueness of $K_{j, A}$ for particular $\partial H_{A} / \partial x_{j}$ will increase considerably in these cases, limiting the length scales for which Darcy's Law remains valid (leaving aside the question whether Darcy's Law is valid at all at a sharp wetting front). This is consistent with Roth's (2008) parabolic relationship between the characteristic time scale of the system and its characteristic length scale.

\section{Illustrative cases}

\subsection{Upscaling the water content-matric head relation- ship at equilibrium and during unit gradient flow}

For the upscaled version of the soil water characteristic $h(\theta)$, the relation $h_{V}\left(\theta_{V}\right)$ is an obvious candidate, but the experimental conditions of $h(\theta)$ need to be taken into account. At the Darcy scale, an undisturbed sample is typically exposed to a fixed $H$ long enough to establish hydrostatic equilibrium (e.g., Dane and Hopmans, 2002a, b, c, d; Romano et al., 2002). From the elevation of the sample with respect to the chosen $x_{3, \text { ref }}$ the value of $h$ at the center of the sample is derived. The total water content of the sample is determined (usually by weighing) to calculate a point $(h, \theta)$ on the $h(\theta)$ curve. The height of the sample (usually $\sim 5 \mathrm{~cm}$ ) is assumed to be too small to let the value of $\theta$ be affected by the vertical gradient of $h$ that counters the gradient in the gravitational potential. 
Even at this small scale, assuming the vertical variation in $h$ to be negligible may not be permitted. Liu and Dane (1995) developed a criterion to test the validity of the assumption for the general two-phase case, involving the densities of the wetting and the non-wetting fluid and the geometry of the experimental set-up. In an elegant analysis, Liu and Dane (1995) demonstrated that the vertical extent of the sample smoothed the $h(\theta)$-curve. An example calculation with a point-scale Brooks-Corey relation (Brooks and Corey, 1964), which features a sharp air-entry value that creates a discontinuity in the curve's derivative, resulted in a curve more similar to van Genuchten's (1980) continuously differentiable shape. They calculated the smooth average curve by numerically treating the sample as a stack of thin slices of soil with identical properties, but with different values of $h$ corresponding to their respective elevations. Averaging the water contents of the slices gave the sample water content.

For larger and possibly irregularly shaped averaging volumes $V$, the vertical extent of $V$ needs to be accounted for in conjunction with porous medium heterogeneity within $V$. The procedure proposed by Liu and Dane (1995) can be adapted by assuming hydrostatic equilibrium within $V$, characterized by uniform $H$ or by $h_{V}$. Applying the hydrostatic equilibrium condition $H=h+x_{3}$ to Eq. (8) gives:

$$
\begin{aligned}
& h_{V}=H-\frac{\int_{V} \theta x_{3} \mathrm{~d} V}{\int_{V} \theta \mathrm{d} V}=H-\int_{-\infty}^{+\infty} x_{3} \int_{A\left(x_{3}\right)} \theta \mathrm{d} A\left(x_{3}\right) \mathrm{d} x_{3} \\
& {\left[\int_{-\infty}^{+\infty} \int_{A\left(x_{3}\right)} \theta \mathrm{d} A\left(x_{3}\right) \mathrm{d} x_{3}\right]^{-1}}
\end{aligned}
$$

where $A\left(x_{3}\right)$ is the horizontal area $\left[\mathrm{L}^{2}\right]$ of $V$ as function of $x_{3}$. Note that either $H$ or $h_{V}$ is sufficient to fully characterize the distribution of $h$ over $V$ if the boundary of $V$ is known. The corresponding average water content is (see Eq. 6):

$\theta_{V}=\frac{1}{V} \int_{-\infty}^{+\infty} \int_{A\left(x_{3}\right)} \theta \mathrm{d} A\left(x_{3}\right) \mathrm{d} x_{3}$

An upscaled $h_{V}\left(\theta_{V}\right)$ relationship according to Eqs. (19) and (20) incorporates spatial heterogeneity and allows $h$ to vary with elevation under hydrostatic equilibrium conditions. The relationship for megascopic $V$ (e.g., a soil layer within a field plot, or an entire field) will be of little use to calculate actual flow, but by comparing the actual $h_{V}$ and $\theta_{V}$ to the equilibrium curve, the deviation from equilibrium can be asserted, and the tendency of $V$ to absorb or release water from or to its surroundings (e.g., the groundwater, or a stream) can be established with a more or less quantitative measure.

During unit gradient conditions (Wagenet, 1986), flow in unsaturated soils is strictly gravity-driven. The matric head is uniform throughout $V$, creating a horizontal hydraulic gradient of zero and a vertical gradient of one. Consequently, the vertical flux density is equal to $-K_{3}$. Variations in local values of $\theta$ arise strictly from soil heterogeneity within a non-layered horizon, because the matric head for which $\theta$ follows from the soil water characteristic is uniform. In these conditions, $\theta_{V}$ is calculated with Eq. (20) as before, but $h_{V}$ is simply equal to $h$ at an arbitrary location in $V$ and can be derived from a single tensiometer reading. Readings from multiple tensiometers over an extended period in time can serve to confirm the prevalence of unit gradient conditions.

\subsection{Megascopically horizontal flow in a container filled with a heterogeneous porous medium}

This is a generalization of the hypothetical case discussed by Gray and Miller (2004), where a horizontal, closed rectangular container of length $L[\mathrm{~L}]$, height $B[\mathrm{~L}]$, and crosssectional area $\Omega\left[\mathrm{L}^{2}\right]$ was filled with a porous medium. Two water-filled reservoirs with a constant water level in contact with the porous medium over the full cross-section at opposite ends at $x_{1}=0$ and $x_{1}=\mathrm{L}$ established fixed hydraulic head boundary conditions. A uniform layer of vertical extent $b$ [L] and porosity $n_{2}$ was sandwiched between uniform layers with porosity $n_{1}$. The strip with porosity $n_{2}$ tilted from the container bottom at $x_{1}=0$ to the top at $x_{1}=\mathrm{L}$ to make the distance $c_{2}[\mathrm{~L}]$ between the vertical midpoint of the layer and the container bottom:

$c_{2}\left(x_{1}\right)=\frac{b}{2}+\frac{x_{1}}{L}(B-b)$

Gray and Miller (2004) derived an area-averaged pressure (equivalent to an averaged $h$ ) over a vertical cross-section using the $\theta$-weighted averaging underlying Eq. (8) above. If the integration in Eq. (8) were carried out over $\Omega$ instead of a volume (as explained below Eq. 9) and the head were converted to a pressure, the resulting equation would be identical to Gray and Miller's. According to the superposition principle (Sect. 2.1) that requires identical averaging manipulations for all components of $H$, the gravitational head $x_{3}$ should have been averaged in the same way to arrive at the correct gradient in the averaged $H_{\Omega}$ (with the subscript indicating the cross-sectional average). For $n_{2}>n_{1}$, the depth interval $b$ centered on $c_{2}$ would overcontribute to the areally averaged $\theta$-weighted $h$. The absence of vertical flow implies that $H$ is vertically uniform. With $x_{3}$ linearly increasing with elevation, $h$ must necessarily decrease with elevation at the same rate according to Eq. (3). The overcontribution of $h$ around $c_{2}$ would be cancelled out by an equally large overcontribution of $x_{3}$ with opposite sign.

Initially, Gray and Miller (2004) did not carry out the averaging of the gravitational head arguing that gravitation plays no part in horizontal flow. However, even in horizontal flow, the gravitational head contributes to $H$. Consequently, the calculated $\partial H_{\Omega} / \partial x_{1}$ was erroneous, resulting in an obviously incorrect non-zero water flux while the water levels in the reservoirs at both ends of the column were the same. 
In the next section of their paper, Gray and Miller (2004) developed an averaging equation for the gravitational potential that is the areal averaging equivalent of Eq. (9) above. The consistency between the averaging manipulations of $h$ and $x_{3}$ was thereby restored, and the correct gradient of $H_{\Omega}$ was obtained.

The corrected equations by Gray and Miller (2004) are only applicable to the specific configuration of their hypothetical set-up, and their methodology rapidly becomes intractable for more complicated set-ups. In contrast, the superposition principle outlined above naturally leads to the generally applicable set of Eqs. (7-9), that reduces to Gray and Miller's (2004) equations for their special case. Thus, consistent application of the superposition principle resolves the paradox discussed by Gray and Miller (2004), and the validity of Darcy's Law for the scale for which Darcy himself formulated it remains unchallenged.

\section{Conclusions}

The relationship between the hydraulic, pressure/matric, and gravitational heads is supported by the thermodynamic interpretation of fluid pressure and the gravitational potential. The superposition property of the heads constituting the hydraulic head translates into a consistency requirement for the upscaling manipulations of all heads. With the added constraint that the amount of energy must be conserved during volume integrations, the superposition property produces a set of consistent upscaling equations.

Application to Darcy's Law leads to a set of criteria that must be met to maintain validity of Darcy's Law under upscaling operations. The criteria are based on the requirement that the area-averaged hydraulic conductivity should not depend on the area-averaged hydraulic gradient. A similar criterion for maintaining uniqueness of the area-averaged hydraulic conductivity remains elusive.

Two illustrative cases demonstrate the usefulness of the volume averaging equations. In one case, an apparent paradox reported in the literature that threatened the validity of Darcy's Law was elucidated by demonstrating that it emerged from a violation of the superposition principle.

Acknowledgement. I thank Majid J. Hassanizadeh for bringing the dual porosity paradox to my attention. I also thank Thomas Gimmi and an anonymous reviewer for their valuable comments.

Edited by: E. Zehe

\section{References}

Bear, J. and Bachmat, Y.: Introduction to modeling of transport phenomena in porous media, Kluwer, Dordrecht, The Netherlands, 1991.

Bertin, H. and Quintard, M.: Two-phase flow in heterogeneous porous media III: Laboratory experiments for flow parallel to a stratified system, Transport in Porous Media, 5, 543-590, 1990.

Bhattacharya, R. N. and Gupta, V. K.: Theoretical explanation of solute dispersion in saturated porous media at the Darcy scale, Water Resour. Res., 19, 938-944, 1983.

Brooks, R. H. and Corey, A. T.: Hydraulic properties of porous media, Hydrol. paper no. 3, Colorado State Univ. Fort Collins, Colorado, USA, 1964.

Brutsaert, W.: Hydrology. An introduction, Cambridge University Press, Cambridge, UK, 2005.

Cressie, N. A. C.: Statistics for spatial data. Revised edition, John Wiley \& Sons, Inc., New York, 1993.

Crapiste, G. H., Rotstein, E., and Whitaker, S.: A general closure scheme for the method of volume averaging, Chem. Eng. Sci., 41, 227-233, 1986.

Dane, J. H. and Hopmans, J. W.: Water retention and storage. Laboratory. General, in: Methods of soil analysis. Part 4. Physical methods, edited by: Dane, J. H. and Topp, G. C., SSSA, Madison, Wisconsin, USA, 675-680, 2002a.

Dane, J. H. and Hopmans, J. W.: Hanging water column, in: Methods of soil analysis. Part 4. Physical methods, edited by: Dane, J. H. and Topp, G. C., SSSA, Madison, Wisconsin, USA, 680-683, 2002b.

Dane, J. H. and Hopmans, J. W.: Pressure cell, in: Methods of soil analysis. Part 4. Physical methods, edited by: Dane, J. H. and Topp, G. C., SSSA, Madison, Wisconsin, USA, 684-688, 2002c.

Dane, J. H. and Hopmans, J. W.: Pressure plate extractor, in: Methods of soil analysis. Part 4. Physical methods, edited by: Dane, J. H. and Topp, G. C., SSSA, Madison, Wisconsin, USA, 688-690, 2002d.

de Rooij, G. H., Kasteel, R. T. A., Papritz, A., and Flühler, H.: Joint distributions of the unsaturated soil hydraulic parameters and their effect on other variates, Vadose Zone J., 3, 947-955, 2004.

Gray, W. G.: A derivation of the equations for multi-phase transport, Chemical Engineering Science, 30, 229-233, 1975.

Gray, W. G.: On the definition and derivatives of macroscale energy for the description of multiphase systems, Adv. Water Resour., 25, 1091-1104, 2002.

Gray, W. G. and Miller, C. T.: Examination of Darcy's Law for flow in porous media with variable porosity, Environ. Sci. Technol., 38, 5895-5901, doi:10.1021/es049728w, 2004.

Gray, W. G. and Miller, C. T.: Consistent thermodynamic formulations for multiscale hydrologic systems: Fluid pressures, Water Resour. Res., 43, W09408, doi:10.1029/2006WR005811, 2007.

Groenevelt, P. H. and Bolt, G. H.: Non-equilibrium thermodynamics of the soil-water system, J. Hydrol., 7, 358-388, 1969.

Harter, T. and Hopmans, J. W.: Role of vadose-zone flow processes in regional-scale hydrology: review, opportunities and challenges, in: Unsaturated-zone modeling. Progress, challenges, and applications, edited by: Feddes, R. A., de Rooij, G. H., and van Dam, J. C., Wageningen UR Frontis Series, Vol. 6, Kluwer Academic Publishers, Dordrecht, The Netherlands, 179208, 2004. 
Hassanizadeh, S. M. and Gray, W. G.: Mechanics and thermodynamics of multiphase flow in porous media including interphase boundaries, Adv. Water Resour., 13, 169-186, 1990.

Hillel, D.: Environmental Soil Physics, Academic Press, San Diego, California, USA, 1998.

Howes, F. A. and Whitaker, S.: The spatial averaging theorem revisited, Chem. Eng. Sci., 40, 1387-1392, 1985.

Jacquin, C. G. and Adler, P. M.: Fractal porous media II: Geometry of porous geological structures, Transport Porous Med., 2, 571596, doi:10.1007/BF00192156, 1987.

Jackson A. S., Miller, C. T., and Gray, W. G.: Thermodynamically constrained averaging theory approach for modeling flow and transport phenomena in porous medium systems: 6. Two-fluid-phase flow, Adv. Water Res. 32, 779-795, doi:10.1016/j.advwatres.2008.11.010, 2009.

Jury, W. A., Gardner, W. R., and Gardner, W. H.: Soil physics, 5th ed., John Wiley \& Sons, Inc., New York, 1991.

Kavvas, M. L.: General Conservation Equation for Solute Transport in Heterogeneous Porous Media, J. Hydrol. Eng., 6, 341-350, doi:10.1061/(ASCE)1084-0699(2001)6:4(341)), 2001.

Kitanidis, P. K.: Groundwater flow in heterogeneous formations, in: Subsurface flow and transport: A stochastic approach, edited by: Dagan G. and Neuman, S. P., Unesco, International Associations of Hydrological Sciences, Cambridge University Press, 83-91, 1997.

Lee, H., Zehe, E., and Sivapalan, M.: Predictions of rainfall-runoff response and soil moisture dynamics in a microscale catchment using the CREW model, Hydrol. Earth Syst. Sci. 11, 819-849, 2007.

Liu, H. H. and Dane, J. H.: Improved computational procedure for retentions of immiscible fluids using pressure cells, Soil Sci. Soc. Am. J., 59, 1520-1524, 1995.

Nordbotten, J. M., Celia, M. A., Dahle, H. K., and Hassanizadeh, S. M.: Interpretation of macroscale variables in Darcy's Law, Water Resour. Res., 43, W08430, doi:10.1029/2006WR005018, 2007.

Quintard, M. and Whitaker, S.: Two-phase flow in heterogeneous porous media: The method of large-scale averaging, Transport in Porous Media, 3, 357-413, 1988.

Quintard, M. and Whitaker, S.: Two-phase flow in heterogeneous porous media I: The influence of large spatial and temporal gradients, Transport in Porous Media, 5, 341-379, 1990a.
Quintard, M. and Whitaker, S.: Two-phase flow in heterogeneous porous media II: Numerical experiments for flow perpendicular to a stratified system, Transport in Porous Media, 5, 429-472, 1990b.

Raats, P. A. C. and Klute, A.: Transport in soils: The balance of mass, Soil Sci. Soc. Am. Proc., 32, 161-166, 1986a.

Raats, P. A. C. and Klute, A.: Transport in soils: The balance of momentum, Soil Sci. Soc. Am. Proc., 32, 452-456, 1986 b.

Renard, P. and de Marsily, G.: Calculating equivalent permeability: a review, Adv. Water Res., 20, 253-278, 1997.

Romano, N., Hopmans, J. W., and Dane, J. H.: Suction table, in: Methods of soil analysis. Part 4. Physical methods, edited by: Dane, J. H. and Topp, G. C., SSSA, Madison, Wisconsin, USA, 692-698, 2002.

Roth, K.: Scaling of water flow through porous media and soils, Europ, J. Soil Sci., 59, 125-130, doi:10.1111/j.13652389.2007.00986.x, 2008.

Vereecken, H., R. Kasteel, J. Vandenborght, and T. Harter: Upscaling hydraulic properties and soil water flow processes in heterogeneous soils: a review, Vadose Zone J., 6, 1-28, 2007.

Veverka, V.: Theorem for the local volume average of a gradient revisited, Chem. Eng. Sci., 36, 833-838, 1981.

van Genuchten, M. T.: A closed-form equation for predicting the hydraulic conductivity of unsaturated soils, Soil Sci. Soc. Am. J., 44, 892-898, 1980.

Wagenet, R. J.: Water and solute flux, in: Methods of soil analysis. Part 1. Physical and mineralogical methods, 2nd ed., edited by: Klute, A., ASA, SSSA, Madison, Wisconsin, USA, 1055-1088, 1986.

Whitaker, S.: Diffusion and dispersion in porous media, AIChE Journal, 13, 420-427, 1967.

Whitaker, S.: Flow in porous media I: A theoretical derivation of Darcy's Law, Transport in Porous Media 1, 3-25, 1986.

Yeh, T.-C. J.: Scale issues of heterogeneity in vadose-zone hydrology, in: Scale dependence and scale invariance in hydrology, edited by: Sposito, G., Cambridge University Press, 224-265, 1998.

Zehe, E., Lee, H., and Sivapalan, M.: Dynamical process upscaling for deriving catchment scale state variables and constitutive relations for meso-scale process models, Hydrol. Earth Syst. Sci., 10, 981-996, 2006, http://www.hydrol-earth-syst-sci.net/10/981/2006/. 